\title{
The Memory of Optimized Dispersion-Managed Periodic Optical Links
}

\author{
P. Serena, A. Orlandini and A. Bononi
}

Università degli Studi di Parma, dept. Ingegneria dell'Informazione, v.le G. Usberti 181/A, 43100 Parma (Italy), email: serena@tlc.unipr.it

\begin{abstract}
We provide a new expression of the pseudo-random sequence length needed for reliable testing of DM periodic optical links with optimized pre-and post-compensation, and show that it scales linearly with bitrate.
\end{abstract}

\section{Introduction}

Reliable simulations and measurements of the performance of dispersion-managed (DM) optical links are based on the transmission of a pseudo-random bit sequence (PRBS) of length $2^{n}-1$, where $n$ must exceed an a-priori unknown integer $m$, i.e., the memory of the DM system. The reason is that the PRBS contains all patterns of $n$ bits, and it must thus be able to reproduce all intersymbol-interference patterns.

Knowing the memory of a DM system is of particular interest in the pseudo-linear regime, where the large accumulated dispersion may impose unpractical large values of $n$ in order to correctly reproduce all possible intra-channel distortions [1]. In [1], the authors proposed a simple phenomenological estimate of $m$, and experimentally validated it at $40 \mathrm{~Gb} / \mathrm{s}$ for return-tozero (RZ) and carrier-suppressed RZ (CSRZ) modulation formats. The formula in [1] is based on the maximum cumulated dispersion where signal power is large enough to generate nonlinear effects, and it predicts a scaling of $m$ with the square of the bitrate $R$.

In this work we show that for an ultralong DM system with optimized pre- and post-compensation and with periodic in-line compensation a different expression for $m$ applies, which scales only linearly with $R$. The formula is based on a linearization of the DM nonlinear Schrödinger equation (DM-NLSE) [2], and is validated against split-step Fourier (SSFM) simulations of a single channel NRZ on-off keying (OOK) signal propagating into a $20 \times 100 \mathrm{~km}$ DM link.

\section{Theory}

Starting from a linearization of the DM-NLSE around a constant wave (CW) solution, in [3] a closed-form expression of the power spectral density of amplified spontaneous emission (ASE) noise at the end of a DM link was provided. The same linearization can be used for studying the propagation of a low extinction ratio OOK signal, seen as a CW plus a small informationbearing field. As in [3], the Fourier transforms $\widetilde{P}(z, \omega)$ and $\widetilde{\theta}(z, \omega)$ of the total field power/phase at coordinate $z$ and frequency $\omega$, are related to their corresponding values at the input of the system $z=0$ by:

$$
\left[\begin{array}{c}
\widetilde{P}(z, \omega) \\
2 P \widetilde{\theta}(z, \omega)
\end{array}\right]=e^{\mathbf{M}(\omega) z}\left[\begin{array}{c}
\widetilde{P}(0, \omega) \\
2 P \widetilde{\theta}(0, \omega)
\end{array}\right],
$$

where $P$ is the average power, and the matrix exponential $e^{\mathbf{M}(\omega) z}$, detailed in [3], contains all the dispersionmanaged system parameters. For an ultralong $N$-span

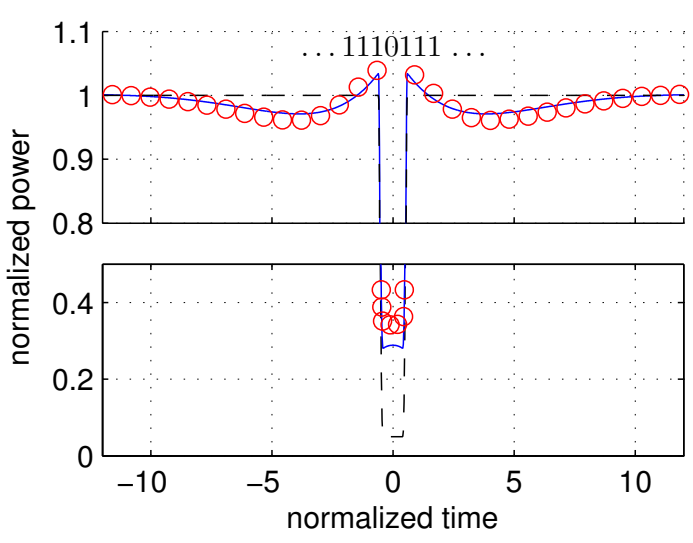

Fig. 1: Received power using SSFM (circles), eq. (1) (solid). Dashed: Tx power. $R=100 \mathrm{~Gb} / \mathrm{s}, \xi_{\text {in }}=0$.

periodic DM system of span-length $L \gg 1 / \alpha$, with $\alpha$ the attenuation, such parameters have been shown to be the nonlinear cumulated phase $\Phi_{N L}=N P \gamma / \alpha$, the map strength $S=\chi \Delta D / \alpha$, and the average cumulated in-line dispersion $\xi_{\text {in }}=\chi N D_{\text {in }}$. In the above expressions, $\gamma$ is the fiber nonlinear parameter; $\chi=$ $(\lambda R / d)^{2} /(2 \pi c)$ is a normalized-to-standard units conversion factor (with $\lambda$ the channel wavelength, $d$ the pulse duty cycle and $c$ the speed of light); and $\Delta D=$ $D_{T X}-D_{\text {in }} / L$ is the deviation of the transmission fiber dispersion $D_{T X}[\mathrm{ps} / \mathrm{nm} / \mathrm{km}]$ from the in-line cumulated dispersion per span $D_{\text {in }}[\mathrm{ps} / \mathrm{nm}]$.

We argue that the simplified model (1) contains all the basic information on the nonlinear propagation of a practical modulated signal in a DM optical system, including the memory of the optical system. Consider for instance a $20 \times 100 \mathrm{~km}$ DM link, with $D_{T X}=17$ $\mathrm{ps} / \mathrm{nm} / \mathrm{km}, \alpha=0.2 \mathrm{~dB} / \mathrm{km}$, full in-line compensation $\xi_{\text {in }}=0$, and no pre- and post-compensation. Fig. 1 shows the response at bitrate $R=100 \mathrm{~Gb} / \mathrm{s}$ and $\Phi_{N L}=$ $0.2 \pi$ [rad] to the sequence ...1110111 .., which well approximates the response to a negative Dirac delta. The dashed line in the figure gives the transmitted power, the solid line the received power predicted by the filter (1), while the circles give SSFM simulations. The figure shows that the presence of the space bit perturbates the power within a time window whose duration is well captured by the linear model (1).

The useful information about the DM system memory must be extracted from the system matrix $e^{\mathbf{M}(\omega) z}$. Using a singular value decomposition (SVD), the system 

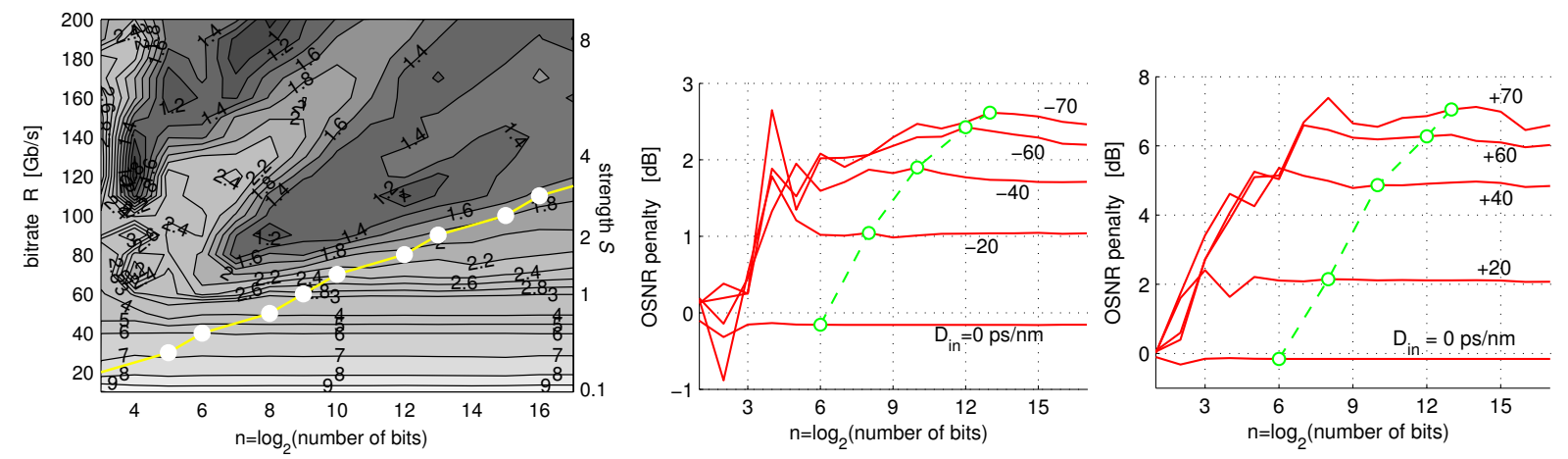

Fig. 2: (Left): Nonlinear threshold $[d B]$ at $1 d B$ of OSNR penalty @ BER $=10^{-5}$ vs. $R$ and $n$ with full in-line compensation. (Center+right): OSNR penalty vs. $n$ for various in-line dispersions @ $R=40 \mathrm{~Gb} / \mathrm{s}$ and $\Phi_{N L}=0.15 \pi$.

matrix can be expressed as $e^{\mathbf{M} z}=\mathbf{U D V}^{T}$, where ${ }^{T}$ indicates transposition, $\mathbf{U}$ and $\mathbf{V}$ are orthogonal matrices that diagonalize $\mathbf{P}=e^{\mathbf{M} z} e^{\mathbf{M}^{T} z}$ and $\mathbf{Q}=e^{\mathbf{M}^{T} z} e^{\mathbf{M} z}$ respectively, while $\mathbf{D}$ is a diagonal matrix whose diagonal elements are the nonnegative square roots of the eigenvalues of $\mathbf{P}$. The geometric interpretation of the SVD is simple: at a specific frequency $\omega$, the transfer matrix acting in the power/phase plane introduces first a rotation through $\mathbf{V}$, then a stretching due to $\mathbf{D}$, and finally a rotation due to $\mathbf{U}$. When the rotations of $\mathbf{U}$ and $\mathbf{V}$ are counteracted by an optimal choice of pre- and post-compensating fibers [4], then the net effect of the nonlinear distortion, and thus the memory, comes from the eigenvalues of $\mathbf{D}$. For small $\Phi_{N L}$, we evaluated the $3 \mathrm{~dB}$ bandwidth of such eigenvalues, and used twice the inverse of the smallest value to get an estimate of the memory of a DM link with optimized pre- and postcompensation as:

$$
m \cong\left\lceil\frac{4 \pi d}{\sqrt{3}}\left(3 S^{2}+\xi_{i n}^{2}\right)^{1 / 4}\right\rceil,
$$

being $\lceil x\rceil$ the smallest integer larger than $x$. Since both $S$ and $\xi_{\text {in }}$ are proportional to $R^{2}$, a noteworthy implication of (2) is that $m$ scales linearly with $R$.

\section{Numerical Checks}

We next check the above theoretical results. Our target was to measure the DM link memory from SSFM simulations by varying either the bitrate $R$ or the in-line dispersion $D_{\text {in }}$ of a single NRZ-OOK channel propagating into a $20 \times 100 \mathrm{~km}$ DM system, with $D_{T X}=$ $8 \mathrm{ps} / \mathrm{nm} / \mathrm{km}$. For the sake of simplicity we overlooked fiber's slope. Once the three system parameters $\Phi_{N L}, S, \xi_{\text {in }}$ have been extracted from the physical parameters, the simulations acquire a much more general meaning [3]. We derive information on memory by measuring the threshold $\Phi_{N L}$ that gives $1 \mathrm{~dB}$ of optical signal-to-noise ratio (OSNR) penalty vs. backto-back at a bit error rate $B E R=10^{-5}$ for increasing PRBS lengths. With this procedure, the nonlinear distortion remains comparable for all cases. We used SSFM simulations for the nonlinear propagation into the fibers and the Karhunen-Loève algorithm for semi- analytical BER estimation. First we checked the impact of strength $S$ by analyzing a fully compensated system. We used $-173 \mathrm{ps} / \mathrm{nm}$ of pre-compensation before transmission and we optimized the post-compensation after transmission for each $R$ in the longest PRBS case, corresponding to $2^{17}=131072$ bits. The propagation inside the pre- and post-fibers were assumed purely linear. Fig. 2(left) gives the nonlinear threshold vs. the PRBS length and the bitrate $R$. The threshold is represented in a dB scale as $10 \log _{10}\left(\Phi_{N L} /(0.1 \pi)\right)$, so that threshold penalties are equal to power penalties. The solid line with circles gives equation (2), and it captures quite well the edge of the stable zone of the contour, i.e. with horizontal levels, where the threshold is independent of the PRBS length.

In the center and right plots of the same figure we fixed $\Phi_{N L}=0.15 \pi$ and $S=0.35$ (i.e. $R=40 \mathrm{~Gb} / \mathrm{s}$ in this case) and measured the OSNR penalty by varying the cumulated in-line dispersion. The center graph refers to $D_{\text {in }}=-70,-60,-40,-20,0 \mathrm{ps} / \mathrm{nm}$, the right graph to $D_{\text {in }}=0,20,40,60,70 \mathrm{ps} / \mathrm{nm}$. Here the pre-compensation was chosen as $-173-\frac{N-1}{2} D_{\text {in }}$ $\mathrm{ps} / \mathrm{nm}$ [4] while the post-compensation was optimized to $223-\frac{N-1}{2} D_{\text {in }} \mathrm{ps} / \mathrm{nm}$. Solid lines refer to SSFM simulations, while dashed circles refer to approximate formula (2). We note that (2) gives a good estimation of DM system memory when the in-line dispersion does not exceed $\pm 50 \mathrm{ps} / \mathrm{nm}$, i.e. when $\left|\xi_{\text {in }}\right| \lesssim 6|S|$.

\section{Conclusions}

We proposed a novel expression for the minimum number of bits required to correctly test the performance of a dispersion-managed optical system. We showed that such a number scales linearly with the bitrate $R$.

\section{References}

1. L. K. Wickham et al., IEEE Photon. Technol. Lett., vol. 16 (2004), pp. 1591-1593.

2. M. J. Ablowitz et al., J. Opt. Soc. Am. B, vol. 19 (2002), pp. 425-439.

3. P. Serena et al., J. Opt. Soc. Am. B, vol. 24 (2007), pp. 773-787.

4. Y. Frignac et al., in Proc. OFC 2002, ThFF5. 